\title{
Téoros
}

Revue de recherche en tourisme

\section{Quelques jalons pour une histoire du tourisme et de la gastronomie en France}

\section{Julia Csergo}

Volume 25, numéro 1, printemps 2006

Entre la culture du goût et le goût de la culture

URI : https://id.erudit.org/iderudit/1071026ar

DOI : https://doi.org/10.7202/1071026ar

Aller au sommaire du numéro

Éditeur(s)

Université du Québec à Montréal

ISSN

0712-8657 (imprimé)

1923-2705 (numérique)

Découvrir la revue

Citer cet article

Csergo, J. (2006). Quelques jalons pour une histoire du tourisme et de la gastronomie en France. Téoros, 25(1), 5-9. https://doi.org/10.7202/1071026ar d'utilisation que vous pouvez consulter en ligne.

https://apropos.erudit.org/fr/usagers/politique-dutilisation/ 


\section{Quelques jalons pour une histoire du tourisme et de la gastronomie en France}

\begin{abstract}
Julia Csergo
Se nourrir est une des premières préoccupations du voyageur. S'il recourt parfois à une nourriture «tirée du sac » (Csergo, 2001), à un "en-cas » que de nombreux récits de voyages mentionnent et à laquelle Guy de Maupassant a donné, dans Boule de suif, une dimension tragique (Montpassant, 1984), le voyageur restaure aussi ses forces dans les auberges, les relais de poste ou chez l'habitant. Aussi, le voyage se prête-t-il particulièrement à la découverte de l'altérité alimentaire. Goûter la production locale, ressource naturelle ou accommodement culinaire, fait partie de la découverte de l'Autre et de l'ailleurs, répond à la quête de ce qui fait l'originalité des régions traversées. Toutefois, penser ainsi l'histoire du déplacement relèverait de l'anachronisme: en effet, contrairement au voyageur contemporain qui ne pratique pas le tourisme sans y associer la dimension gastronomique, qui va jusqu'à entreprendre un déplacement en quête de la spécialité alimentaire locale, qui consomme forcément des andouilles à Guéméné, du pain d'épices à Dijon ou du cassoulet à Castelnaudary, longtemps les voyageurs ont ignoré le détail des habitudes alimentaires, des goûts régionaux et des savoir-faire culinaires des contrées traversées.
\end{abstract}

\section{Du « goût » du voyage à la « monumentalisation » de la spécialité alimentaire}

Durant le XVIIe siècle, l'élite qui relate ses voyages, convaincue sans doute de la primauté de la cuisine de cour, ne s'intéresse que peu à la cuisine du peuple des villes et des campagnes. Son regard s'attarde plutôt sur le paysage. Au mieux, elle peut porter une appréciation, étonnée ou navrée, sur

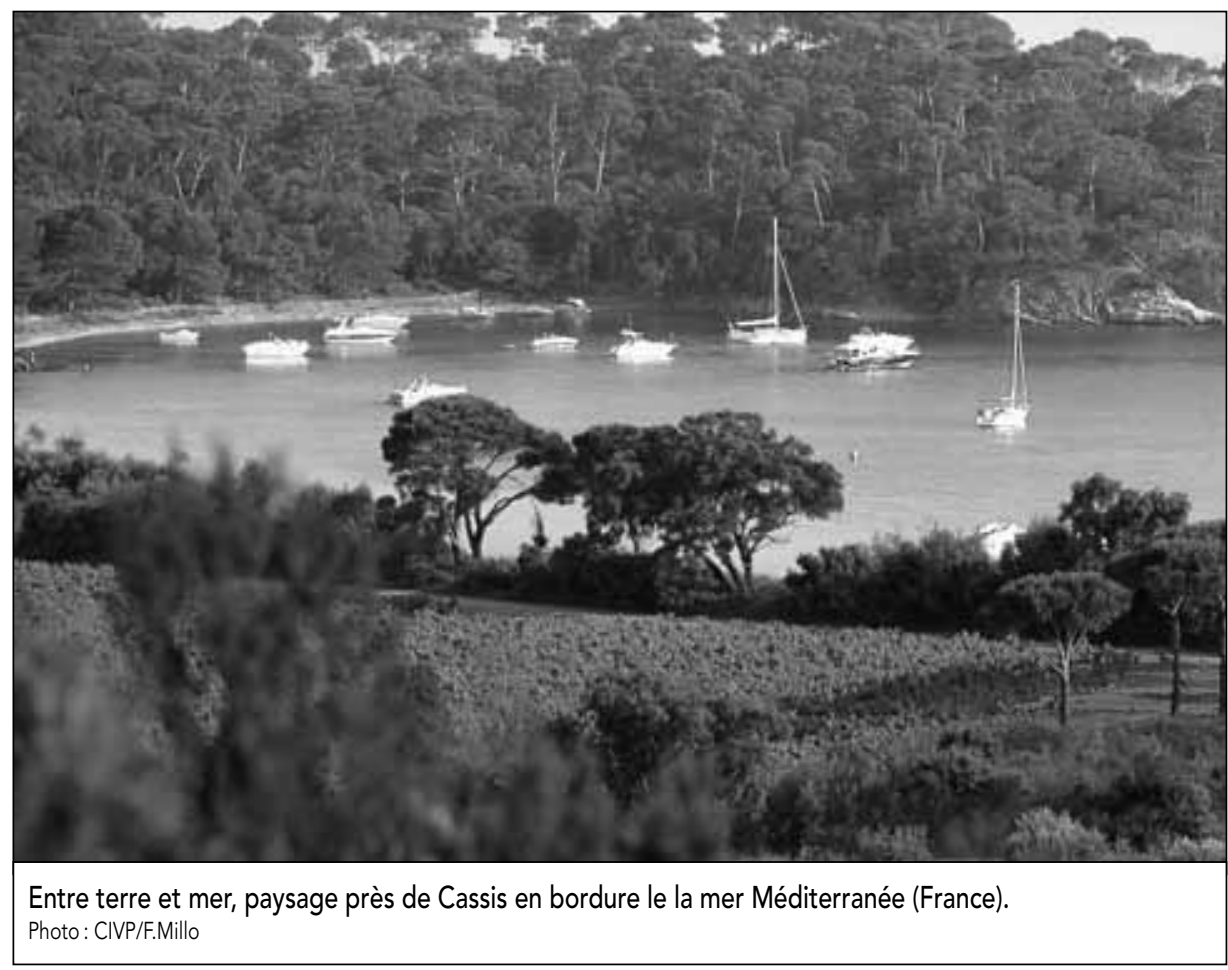

la bonté ou la médiocrité de la chère servie dans les auberges ou colporter des généralités sur l'abondance ou la rareté des denrées dans telle région. Ainsi, pour la Provence (Csergo, 1998), par exemple - excepté Jean-Jacques Bouchard qui s'étend, lors de son voyage effectué en 1630, mais publié en 1881 (Bouchard, 1881), sur les coutumes culinaires des Provençaux -, les voyageurs, gourmands de paysages avant tout, ne s'attardent quasiment pas sur les cultures banales, comme celle du blé, pour ne s'attacher qu'aux spécialités visibles dans le paysage et dotées d'un statut symbolique élevé: les arbres fruitiers et les herbes odoriférantes pour ce qui concerne le domaine végétal ; les perdreaux, les becfigues, les ortolans pour les espèces animales. Des orangers et des citronniers, abondamment mentionnés, ils n'évoquent jamais le goût acide ou les usages alimentaires, mais seulement le parfum ; les fruits confits ne sont mentionnés qu'à travers l'anecdote qui rapporte qu'en 1660, pour faire honneur à la visite de Louis XIV, le chevalier de Saumur fit confire sur ses orangers mêmes une partie des oranges. Si les oliviers retiennent leur attention, ils ne s'arrêtent jamais à l'usage alimentaire des olives et de I'huile d'olive, non plus qu'aux aliments dotés d'un statut inférieur, l'ail par exemple, dont on sait par ailleurs qu'il est alors particulièrement prisé en Provence et dans tout le Languedoc. Ils mentionnent rarement les accommodements culinaires spécifiques d'une région, comme si l'évocation de l'aliment et de la cuisine se voyait entachée d'une certaine "malséance ». Quelqu'un comme $\mathrm{M}^{\mathrm{me}}$ de Sévigné ne relate jamais, par le menu, ce genre de quotidienneté, dont elle 
excelle pourtant à rapporter, pour sa santé ou ses repas de malade, les infimes détails (Sévigné, 1978).

Dans la seconde moitié du XVIII e siècle, le regard du voyageur se modifie sous l'influence de divers facteurs. Les avancées des techniques des Ponts et Chaussées permettent une meilleure exploration du territoire. Dans le même temps, alors que la formidable croissance urbaine forge, notamment avec l'essor du mouvement romantique, de nouvelles représentations de la nature, du monde rural et de la saine rusticité du peuple des campagnes, s'affirme et se précise le regard ethnographique sur la province et sur ses coutumes particulières. À un moment où la réflexion sur les origines traverse la réflexion intellectuelle, l'espace local devient objet de savoir et de mémoire. Désormais, les récits de voyages veilleront à rapporter la diversité et la spécificité des lieux, des hommes, des mœurs aussi parmi lesquels figureront les coutumes alimentaires locales. Ainsi, dans la relation du voyage qu'il entreprend de 1775 à 1807, François Marlin écrit : « Je conviens qu'un voyageur ne doit pas tenir le registre de ses repas et de ses auberges ; mais ces détails ne sont pas toujours superflus [...] Une anecdote de cabaret [...] peut donner une idée juste des mœurs d'une ville, et même d'un pays. » (Marlin, 1817)

Même s'il se fait parfois sous forme de dénonciation des archaïsmes, l'intérêt pour la coutume culinaire locale apparaît plus ouvertement: dans les années 1760-1800, le compagnon Ménétra, retraçant son tour de France, s'attarde sur les " petits pieds " (probablement pieds et paquets) qu'on lui sert à l'hôpital de Montpellier, sur la soupe à l'huile qu'on lui offre à Lyon, sur la soupe aux harengs à laquelle il refuse de goûter à Romorentin (Roche, 1982). Parmi tous les récits de voyages en France que compte la décennie révolutionnaire, citons Goethe qui découvre, en 1792, le "pot-au-feu » chez des riches paysans Argonnais (1933:144145) ; Grasset de Saint-Sauveur qui signale, à propos des paysans landais qu'il rencontre en 1797 : «Leur repas est frugal : il consiste en un morceau de cruchade qu'ils trempent dans un peu de sauce extraite du jus du lard" (1988: 57); l'Anglais Arthur Young qui découvre, lors de son voyage en France (1787-1789), une cuisine, dit-il, « infiniment supérieure à la nôtre » et qui goûte le «Schnitz » à Strasbourg : « un plat de jambon (lard) et de poires cuites. On dirait un mets pour le dîner du diable, mais en le goûtant, je fus surpris de le trouver passable » (1976 : 346) ; ou encore Saint-Amans, en 1789, qui raconte à Héas, dans les Pyrénées, la confection de la soupe quotidienne chez des paysans (2003: 73-74).

Néanmoins, le véritable tournant qui annonce le changement durable de statut de la particularité alimentaire telle qu'elle s'inscrit dans la localité et dans la relation au voyage s'effectue à la fin du XVIII ${ }^{e}$ siècle. À cette époque, sous l'influence des nouveaux gastronomes et de leurs discours, les savoir-faire alimentaires et culinaires deviennent un art (Ory, 1998). Les premières entreprises de recensement des spécialités locales se développent, alors que s'élabore la première cartographie gourmande du territoire conçue selon le vœu de Grimod de la Reynière (1807), sur le principe de ce qui deviendra la carte touristique: "Ainsi, au lieu du clocher d'Amiens, on distinguerait à cette place un pâté de canard; à Nérac, une terrine de perdrix rouges; une de foies de canards à Toulouse ; des pieds et du fromage de cochon à Troyes ; un pot d'épine-vinette à Dijon ; des figues à Ollioules. " Cette carte sera réalisée dans le Cours gastronomique de Cadet de Gassicourt (1809) qui figure, sous forme de bouteilles, de volailles, de poissons, de ruches ou de terrines, les principales productions alimentaires dont les lieux tirent leur célébrité. Aussi, la spécialité gastronomique devient-elle, durablement depuis, un monument lié à un site. Parallèlement, avec l'essor de l'intérêt pour l'ethnographie, la coutume locale devient le creuset de la mémoire des ancêtres. Dans ce mouvement, l'enracinement mémoriel de la spécialité gastronomique apparaît, dès le début du XIXe siècle, à travers la production de livres de cuisine régionaux - Alsace (1811), Provence (1830 et 1850) -, puis, plus tardivement, d'ouvrages qui recueillent les traditions alimentaires et culinaires locales et inaugurent le processus de valorisation patrimoniale d'un pays autour de sa table - Alsace (1862), Bresse (1882). Par le biais des commentaires historiques, la gastronomie s'enracine dans des communautés, des localités et des paysages habités par le temps, se construit culturellement dans le passé. À ce titre, elle se verra acquérir progressivement le statut de bien culturel mineur que lui reconnaissent aujourd'hui les économistes du tourisme.

\section{La gastronomie comme nouvelle forme de consommation touristique d'espace local}

Les guides touristiques, qui paraissent en série dès les années 1840, avec l'essor des chemins de fer et des voyages en train, rendent compte des nouvelles formes d'appréhension des territoires par une codification du regard porté et à porter sur la France et ses richesses : ils fixent ainsi un cadre descriptif qui s'ordonne autour de la géographie physique, de l'histoire, des coutumes, des édifices monumentaux, des loisirs et des plaisirs (Chabaud et al., 2000). II ne s'agit pas ici d'analyser la structure de chacune de ces séries, mais force est de constater que ces guides signalent, de façon de plus en plus systématique et ordonnée, la spécialité alimentaire locale.

Prenons l'exemple des guides Joanne. Pour chaque localité traversée, la notice détaille les éléments les plus remarquables sur lesquels le touriste doit orienter ses pas et poser son regard. C'est à la fin de ces notices qu'il peut parfois, très ponctuellement dans un premier temps, être fait mention des «industries et produits particulièrement intéressants ", où l'on trouve, pêle-mêle, productions industrielles et agricoles, produits du commerce local, ressources alimentaire naturelles - poissons, gibiers - ou agricoles - céréales, fruits. L'édition de 1877 indique, de Bourg en Bresse, que la ville fait un commerce actif de volailles de Bresse; de Grenoble, l'industrie du Saint-Marcellin et celle des liqueurs de la Chartreuse - Genepy, Ratafia, China - ; de Lyon, aucunement la réputation gastronomique, mais les produits chimiques, la fabrication de machines, les soieries. Plus précise, l'édition de 1883 signale de Périgueux les farines, les vins, les eaux de vie, les bestiaux, les gibiers, les volailles, les truffes et les «pâtés truffés dont la renommée est européenne ». Ainsi, la production alimentaire locale n'est indiquée que dans la perspective de l'inventaire des richesses industrielles et de la prospérité commerciale de la région : la ressource locale, y compris alimentaire, est avant tout une ressource économique dont la dimension culturelle et succulente est absente; la gastronomie ne fait pas encore partie de l'économie touristique.

Les premiers Guides bleus ne se distinguent pas des Joanne qu'ils reprennent parfois intégralement. Mais, progressivement, la présentation de la région se fait plus érudite, plus ethnographique; les activités hu- 


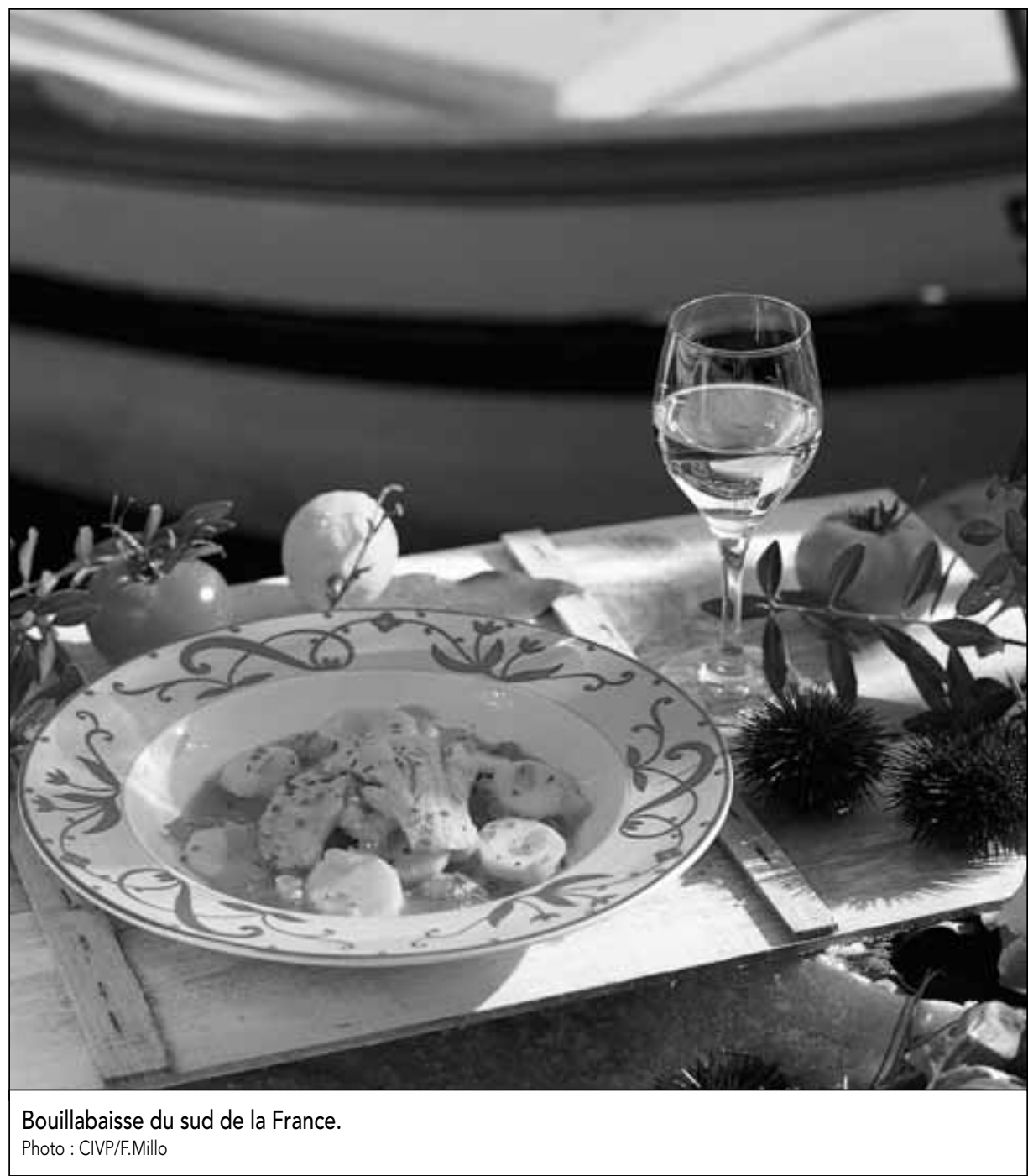

maines englobent désormais les parlers, les folklores, les légendes, les costumes. À partir des années 1920, la rubrique « industries et produits particulièrement intéressants " commence à opérer une distinction entre produits locaux industriels et agricoles et signale parfois la spécialité culinaire. Le premier cas semble concerner le guide de Savoie de 1922 : pour Annecy, par exemple, il signale "les poteries artistiques, les chocolats, les fromages (reblochon, vacherin) » et, une ligne au-dessous, sous l'intitulé "mets", une nouvelle rubrique: "Gratin de pommes de terre, rissoles, truites, ombles chevaliers, vins ». La même année, le guide Bords de Loire et Sud-Ouest intègre, dans la présentation générale introductive, un chapitre consacré aux « Industries intéressantes pour les touristes, spécialités gastronomiques et principaux crus » : on y trouve les produits de l'industrie alimentaire - charcuteries, fromages, confiseries - ainsi que les plats régio- lité locale signalée par le guide, aussi pittoresque que le paysage auquel elle s'associe. Les grands voyageurs n'avaient pas omis cette dimension, tel Auguste Bleton qui résumait : "Plus ce qu'on vous sert diffère de ce que vous mangez d'ordinaire, plus vous êtes en voyage. » (1899: 5)

\section{Les acteurs d'une «Sainte Alliance "}

Dès le début du $X X^{e}$ siècle, en liaison avec l'essor des mobilités individuelles, le vélo et l'auto, l'espace des voyages s'est ouvert aux "obscurs recoins" non desservis par les chemins de fer. Bien que l'automobile soit à l'époque un sport mondain, les potentialités qu'elle ouvre annoncent l'essor d'un nouveau secteur économique, propice au développement local : l'industrie du tourisme qui intéresse autant l'industrie automobile que celles de l'hôtellerie et de la restauration. De puissantes associations comme le Touring Club de France et l'Automobile Club de France s'y impliquent efficacement. D'emblée, la gastronomie se voit associée à la pratique touristique. En 1906, le Touring Club écrit dans sa revue:

On ne saurait trop encourager les hôteliers de province à garder la tradition des plats de pays, des condiments de la contrée, bien autrement affriolants pour la grande majorité des touristes que les sauces Béchamel, Colbert et Sévigné qui poursuivent les voyageurs de leurs arômes fadasses et quelconques d'un bout à l'autre du monde habité.

let à la rabelaisienne " pour Touraine ou la « langue de bœuf en gelée » pour Anjou.

Ainsi, très visiblement, ce sont les années 1920 qui inaugurent, de façon durable, les rubriques gastronomiques dans les guides touristiques imprimés. Quelques années plus tard, ce sont de pleines pages qui seront consacrées, dans l'introduction générale cette fois, à la présentation gastronomique de chaque région : en 1931, quatre pages traitent de la gastronomie de la Bourgogne, de la Franche-Comté ou du Lyonnais - «qui jouit d'une réputation mondiale en matière gastronomique ». Désormais, la gastronomie vient s'agréger aux autres objets patrimoniaux, points de vue, édifices, personnages illustres, qui ponctuent les paysages locaux. Le voyageur curieux de connaître l'Autre et l'ailleurs se doit donc de rechercher un dépaysement alimentaire, de goûter la spécia-
De multiples initiatives suivent. Celle qu'entreprend le Club des Cent, fondé en 1912 par des «gourmets émérites » et des «touristes convaincus » souhaitant « faire œuvre de propagande gastronomique et touristique ", est à l'origine de nombreuses actions qui se déploieront surtout dans les années 1920 (Csergo, 2006). Citons les principales : celle du Michelin, guide fondé en 1901 pour les automobilistes, qui publie en 1914, sous forme de cartographie, les «Spécialités locales à manger sur place ou à emporter » et indique les restaurants recommandés et leurs spécialités; celle du Touring Club qui, caressant l'ambition de réaliser un inventaire des spécialités régionales, publie en 1920 une carte des spécialités régionales et une rubrique intitulée "Nos richesses gastronomiques "; celle de l'Automobile Club qui lance en 1921 dans les régions le "Concours de la bonne 
cuisine "; celle encore de l' "Association des syndicats des agences de voyage " qui, en 1921, publie dans son organe officiel les "Voyages autour de la table ", chroniques de gastronomie régionale; celle enfin de Rouff et Curnonsky qui inaugurent en 1921, avec leur Tour de France gastronomique publié en 24 volumes, un nouveau genre de guide touristique, le "guide gastronomique ", dont on connaît aujourd'hui la fortune. Inventoriés région par région, produits locaux, recettes typiques, meilleurs restaurants de spécialités, dessinent, des années 1920 aux années 1950, une nouvelle carte touristique de la France. La consommation de spécialités alimentaires locales se conçoit désormais en liaison avec la consommation de sites et de monuments. En 1913, alors que s'annonce la grande Loi sur la protection des monuments historiques, Louis Forest, président du Club des Cent, annonce le processus de monumentalisation qui affectera les gastronomies régionales en engageant le pays à "sauvegarder nos trésors d'art alimentaire comme d'autres veillent sur les monuments ${ }^{1}$. En 1923, dans un guide intitulé Où déjeunons-nous, Gaston Derys déclare: "Le voyage en France n'est pas seulement un pèlerinage à travers les musées, les palais, les châteaux, les cathédrales : c'est aussi, pour tout homme de goût, une exploration sans cesse renouvelée à travers les merveilles culinaires de notre pays. » Aussi, par l'intermédiaire de multiples circuits, carnets de routes, randonnées, promenades, vagabondages gastronomiques, l'édifice monumental, la curiosité naturelle, le paysage, la gloire locale, ces éléments qui symbolisent et identifient un site ou une région, s'imposent-ils désormais comme étant la truffe, le pâté de foie, la poularde, les gaudes... La relation à l'histoire nationale qui ponctue les itinéraires du « mangeur de routes » ne se fait plus par le biais de la référence à une entrée royale ou à la signature d'un traité, mais par celui du rôle que jouait sous l'Ancien Régime à Périgueux, par exemple, l'offrande de pâtés de foie aux intendants. Les «lieux» gastronomiques deviennent aussi prestigieux que Bouvines ou Alésia... Alors que l'aventure touristique s'installe dans un rattachement fervent et savoureux à la terre, le touriste s'avance vers des lieux reconnaissables, dans l'attente des particularités que les guides lui annoncent, lui mettent en scène, lui mettent en bouche.

Au début du XXe siècle, l'essor de l'activité touristique conduit les régions, notamment à travers le mouvement régionaliste dont le réveil s'opère au début du XXe siècle, à s'associer à la démarche et à utiliser les cultures régionales comme faire-valoir (Thiesse, 1991). À cet égard, le chantre et le théoricien du régionalisme, Jean Charles-Brun, avait rédigé, à la fin du siècle, pour le Touring Club, une brochure intitulée Tourisme et Régionalisme (1890) : son but était de montrer les liens susceptibles d'être entretenus entre l'idéologie régionaliste et l'activité touristique, cette dernière trouvant, dans le maintien et la revendication des diversités régionales, un terrain favorable à son développement. Ici, grâce à la mise en valeur du local, le tourisme apparaît non seulement comme un auxiliaire du programme régionaliste, mais encore comme un dessein patriotique. Si les militants régionalistes semblent initialement méfiants vis-à-vis du dévoiement des cultures locales que peut induire leur exhibition touristique, ils finissent néanmoins par s'investir dans l'action touristique, voyant là un moyen de se réapproprier les cultures traditionnelles. On peut se référer à ce propos à la série de guides touristiques régionalistes de Van Bever et Van Genep, La France pittoresque et artistique, dont le premier volume paraît en 1913: leur but avoué est d'attirer l'attention des touristes sur des régions et des sites mal connus, de mettre en lumière l'histoire, les mœurs, les coutumes et les traditions locales ainsi que les écrivains qui se sont employés à les exalter. Ici, la table est évoquée à travers des traditions gastronomiques, des spécialités alimentaires, des recettes locales tirées autant de livres de cuisine que d'une littérature et d'une poésie gastronomique, un genre ancien vantant le cidre, le brie, les tripes à la mode de Caen, etc. À la fin de chaque volume, un cahier pratique signale, sous le titre «Ce qu'il faut voir, ce qu'il faut savoir ", les spécialités gastronomiques de chaque localité. Toutefois, force est de constater que, si l'on se réfère aux programmes de fêtes régionalistes organisées à la même époque dans plusieurs localités, au regard des autres "marqueurs identitaires " - pour reprendre ici la terminologie de nos collègues ethnologues -, les spécialités alimentaires n'apparaissent que de façon secondaire par rapport aux danses, aux costumes et aux langues locales.

Dans le souci d'animer le pays et de vivifier le tissu économique local, ce sont encore les professionnels de l'hôtellerie et de la restauration qui rejoignent le mouvement. Les congrès d'hôteliers concentrent leur attention sur le maintien des traditions culinaires et gastronomiques locales, allant jusqu'à prôner, comme l'avait fait le Félibrige de Mistral, association de défense de la langue et de la culture provençales, l'appellation des plats locaux dans les langues régionales. Dans sa brochure, Charles-Brun (1890) note à cet égard que «l'intérêt bien compris a mué en d'excellents régionalistes de simples hôteliers désireux de faire participer leurs belles contrées de France à l'extraordinaire développement du tourisme". En 1921 est ouverte la première «Foire gastronomique de Dijon » qui commercialise les spécialités de pays, gloires de bouche souvent mythiques comme la côte d'agneau à la "Téméraire ", la cassolette de grives Jean-sans-Peur ou le poulet sauté des ducs de Bourgogne... En 1923, à Paris, le Salon d'Automne introduit dans ses manifestation l'art culinaire régional promu au rang de "neuvième art » et reconnu comme " essentiel au tourisme et au renom extérieur de la France ". À cet égard, le salon propose une exposition-dégustation de spécialités de seize régions représentées chacune par un chef local installé dans des décors « régionaux » recréés à cette fin par le sculpteur Temporal. L'ambition de cette exposition est clairement annoncée : "Gourmets, si vous n'êtes touristes, vous le deviendrez rien qu'à avoir goûté à ces bonnes choses des bons terroirs de France. " Le régionaliste Charles-Brun y intervient encore :

Chaque province de France a son parler, sa sensibilité particulière, sa littérature et son art; elle possède son tour de main, des vieilles recettes, sa tradition gastronomique [...] On ne forcera pas les méridionaux à s'empiffrer de beurre ni les gens du Nord à s'arroser d'huile. (Salon d'Automne, 1923)

Toutefois, nombreux sont les acteurs qui témoignent de la difficulté à susciter l'enthousiasme des restaurateurs vis-à-vis des cuisines locales. En 1919, Louis Forest, président du Club des Cent dont nous avons déjà évoqué l'engagement dans le développement du lien tourisme et gastronomie, signale, lors d'un voyage en Alsace:

Je tiens à noter la tendance fâcheuse des maisons les plus recommandables à oublier de façon systématique les qualités de leur cuisine locale [...] Ayant fait comparaitre devant moi de notables restaurateurs de Strasbourg, je ne tardai pas à comprendre que je les vexais en leur demandant les plats et les vins du pays. 
L'un d'eux me répondit aigrement que l'on faisait le Chateaubriand aussi bien chez lui qu'à Paris et qu'il ne fallait pas le prendre pour une auberge de pays [...] Un autre m'affirma que sa cave était assez garnie pour pouvoir se passer des vins d'Alsace [...] Un troisième qu'il était inutile de compliquer la cuisine avec des spécialités du pays qui coûtent cher et auxquelles la foule de touristes étrangers n'entend rien².

En effet, le discours de la centralité, de Paris, ne trouve pas immédiatement d'écho dans les régions. En 1921, par exemple, la correspondante "Limousin » de la revue du Touring Club attire l'attention des promoteurs des spécialités locales sur le fait que beaucoup de spécialités alimentaires locales, trop éloignées des exigences de la gastronomie parisienne, deviennent «immangeables " par les touristes : elle donne l'exemple du "clafoutis avec ses fruits qui ont conservé noyaux et queues ", du "galetou de blé noir au goût d'éponge et de glaise $[\ldots] »$.

Quelques années plus tard, dans son entreprise de valorisation touristique des gastronomies régionales, pour laquelle il lancera la formule «Sainte Alliance du tourisme et de la gastronomie », Curnonsky s'attachera à établir une distinction, salutaire pour la consommation touristique, entre les spécialités alimentaires locales susceptibles de s'intégrer dans la cuisine nationale, parce qu'elles "sont accessibles au commun des mortels" - le confit et le foie gras du Périgord par exemple -, et les spécialités " inassimilables par les estomacs nationaux [...] parce qu'il faut une longue hérédité pour pouvoir les absorber » - comme la cruchade ou la garbure. Une réserve qui se verra en grande partie estompée par la reconnaissance des patrimoines coutumiers - patrimoines socio-ethnographiques, un processus qui s'achève avec l'invention du patrimoine rural - des architectures et des paysages aux savoir-faire et aux produits du terroir ${ }^{3}$ - et qui verra, dans les années 1990, la gastronomie accéder, en France, sous l'égide des ministères de la Culture, de l'Agriculture et du Secrétariat d'État au Tourisme, au statut d'objet patrimonial indissociable des sites, des monuments et des procédures d'accueil des touristes (Csergo, 1997).

\section{Conclusion}

Si goûter la nourriture locale s'impose comme une activité touristique incontournable, répondant à la fois à la curiosité cognitive, à la quête de dépaysement, au goût de l'Autre et de l'ailleurs, les cultures alimentaires régionales constituent un enjeu décisif pour une France désireuse d'exporter l'image de ses visages régionaux et soucieuse de maintenir, notamment par la consommation touristique d'identités locales, l'équilibre d'un tissu rural fortement compromis.

Julia Csergo est professeur d'histoire contemporaine à l'université Lumière-Lyon 2.

\section{Notes}

1 Le Matin, mai 1913.

2 Bulletin du Club des Cent, 30 septembre 1920.

3 Voir à ce propos le rapport d'Isaac Chiva (Ministère de la Culture et de la Francophonie, 1994).

\section{Bibliographie}

Outre les diverses éditions des guides touristiques et gastronomiques mentionnés dans le texte:

Bleton, Auguste (1899), Au-delà des Pyrénées. Notes et impressions, Lyon, A. Storck.

Bouchard, Abbé Jean-Jacques (1881), Les Confessions de Jean-Jacques Bouchard, parisien, suivies de son voyage de Paris à Rome en 1630, Paris, Isidore Lisieux.

Cadet de Gassicourt, Charles-Louis (1809), Cours gastronomique ou les diners de Manant-Ville, Paris, Capelle et Renaud.

Chabaud, Gilles, Evelyne Cohen, Natacha Coquery, et Jérôme Penez (2000) (dir.), Les Guides imprimés du XVI au XXe siècle. Villes, paysages, voyages, Paris, Belin.

Charles-Brun, Jean (1890), Tourisme et Régionalisme, Revue du Touring Club de France.

Csergo, Julia (1997), « La constitution de la gastronomie comme objet patrimonial en France", dans Daniel Grange et Dominique Poulot (dir.), L'Esprit des lieux. Le patrimoine et la cité, Grenoble, Presses universitaires de Grenoble, p.183-193.

Csergo, Julia (1998), "Tables provençales au $\mathrm{XVII}$ siècle. Spécialités locales et régionalisme alimentaire ", dans Gilbert Garrier (dir.), Boire et Manger au XVII siècle, Université du vin de Suze la Rousse, p.111-136.

Csergo, Julia (2001) (dir.), Casse-croûte. Aliment portatif, repas indéfinissable, Paris, Éditions Autrement, coll. " Mutations ».
Csergo, Julia (2006) «Du discours gastronomique comme propagande nationale. Le Club des Cent 1912-1930", dans Denis Saillard et Françoise Hache-Bissette, Gastronomie et identité culturelle française. Discours et représentations, $X I X^{e}-X X \mid{ }^{e}$ siècle, Paris, Nouveau Monde.

Goethe, Johann Wolfgang von (1933), Campagne de France et siège de Mayence, Paris, Éditions Montaigne.

Grasset de Saint-Sauveur, Jacques (1797-1798) (1988), Les Landes de Bordeaux: Mours et usages de leurs habitants, préf. de Guy Latry, Bordeaux, éditions Ultreïa.

Grimod de la Reynière, Alexandre Balthazar Laurent (1807), Almanach des Gourmands. Cinquième année, Paris, Maradan.

Marlin, François (1817), Voyages d'un Français, depuis 1775 jusqu'à 1807, Paris, Guillaume, 4 vol.

Maupassant, Guy de [1880] (1984), Boule de Suif, Paris, Le livre de poche.

Ministère de la Culture et de la Francophonie (1994), Une politique pour le patrimoine culturel rural, rapport présenté par Isaac Chiva à Jacques Toubon, Paris, Ministère de la Culture et de la francophonie.

Ory, Pascal (1998), Le Discours gastronomique français des origines à nos jours, Paris, Gallimard/Julliard.

Roche, Daniel (1982), Journal de ma vie: JacquesLouis Menetra, compagnon vitrier au $18^{\mathrm{e}}$ siècle, Paris, Éditions Montalba.

Saint-Amans, Jean-Florimond de Boudon de (2003), Fragments d'un voyage sentimental et pittoresque dans les Pyrénées, Éditions Monhélios, coll. «Les grands classiques du pyrénéisme".

Salon d'Automne (1923), Le livret d'Or de la section gastronomique régionaliste, Paris.

Sévigné, Madame de (1978), Correspondance, Édition critique par Roger Duchêne, Gallimard, Bibliothèque de la Pléiade, Paris, tome III, $1888 \mathrm{p}$.

Thiesse, Anne-Marie (1991), Écrire la France. Le mouvement littéraire régionaliste de langue française entre la belle époque et la Libération, Paris, Presses universitaires de France.

Young, Arthur (1976), Voyage en France 1787, 1788, 1789, Paris, Armand Colin. 